\title{
A COUNTEREXAMPLE CONCERNING THE MAXIMUM AND MINIMUM OF A SUBHARMONIC FUNCTION
}

\author{
ALEXANDER FRYNTOV
}

(Communicated by Albert Baernstein II)

Abstract. For every $\Delta>0$ a function $u$ subharmonic in the plane is constructed such that $u$ has the order $\rho=1+\Delta$ and satisfies the condition

$$
\min _{\varphi} u\left(r e^{i \varphi}\right) / \max _{\varphi} u\left(r e^{i \varphi}\right) \leq-(C+1) \quad \text { for every } r>0 \text {, }
$$

where $C=C(\rho)>0$. This example answers a question of W. K. Hayman.

Let $f$ be an entire function of finite order $\rho$, and let

$$
M(r, f)=\max _{\varphi}\left|f\left(r e^{i \varphi}\right)\right|, \quad \mu(r, f)=\min _{\varphi}\left|f\left(r e^{i \varphi}\right)\right| .
$$

The well-known $\cos \pi \rho$-theorem asserts that if $\rho \leq 1$, then

$$
\limsup _{r \rightarrow \infty} \frac{\log \mu(r, f)}{\log M(r, f)} \geq \cos \pi \rho .
$$

In 1952 Hayman [H2] proved that for every $\rho>\rho_{0}, \rho$ being positive and large enough, there exists an entire function of order $\rho$ such that

$$
\limsup _{r \rightarrow \infty} \frac{\log \mu(r, f)}{\log M(r, f)}<-c(\rho)<-1 ;
$$

in fact, $c(\rho)$ is unbounded as $\rho \rightarrow \infty$. However, the question [H1] of what happens when $\rho$ is close to one remained open up to the present time. The purpose of this paper is to give a particular answer to this question.

In fact, for every $\rho>1$ we construct a function $u$ subharmonic in $\mathbb{C}$ that satisfies the conditions:

(i) $u(0)=0$;

(ii) $B(r, u)=r^{\rho}, 0<r<\infty$;

(iii) $L(u, r) / B(u, r)<-(1+C(\rho))$ for every $r>0$ and some $C(\rho)>0$. (Here $L$ and $B$ denote the lower and upper bounds of $u(z)$ on the circle $|z|=r$.)

In order to get a corresponding example of an entire function, it is sufficient to use a suitable theorem on the approximation of a subharmonic function by

Received by the editors June 12, 1992 and, in revised form, November 3, 1992.

1991 Mathematics Subject Classification. Primary 31A05; Secondary 30C75.

Key words and phrases. Subharmonic functions, entire functions, positive harmonic function. 
the logarithm of modulus of an entire one (see $[\mathrm{A}]$ or $[\mathrm{Yu}]$ ). The details will be given in Appendix 2.

\section{TWO LEMMAS}

To construct this example we need two lemmas.

Lemma 1. For every $T>1$ and every $\tau, T>\tau>1$, there exists a subharmonic function $g$ such that for some $\alpha>0$ the following relations are valid:

$$
\begin{gathered}
B(g, r)=g(-r)=r, \\
g(r)<-(1+\alpha) r \quad \forall r \in \bigcup_{k=-\infty}^{\infty}\left[T^{k}, \tau T^{k}\right]=\mathscr{R}^{+}(T, \tau) .
\end{gathered}
$$

Proof. First we introduce some notation. Let $T, \tau$ be positive numbers such that $1<\tau<T$, and let $I_{\epsilon}$ be the complex $\epsilon$-neighborhood of the interval $I=I_{0}=[1, \tau]$. Let

$$
\mathscr{R}_{\epsilon}(T, \tau)=\bigcup_{k=-\infty}^{\infty} T^{k} I_{\epsilon} .
$$

Denote by $\mathscr{U}_{\epsilon}$ the family of subharmonic functions that satisfy the inequality $\left(z=r e^{i \theta}\right)$

$$
u_{\epsilon}\left(r e^{i \theta}\right) \leq \begin{cases}r(1+\cos \theta) & \text { for } z \notin \mathscr{R}_{\epsilon}(T, \tau), \\ 0 & \text { for } z \in \mathscr{R}_{\epsilon}(T, \tau) .\end{cases}
$$

Let $u$ be the least upper bound of this family. Obviously, $u$ is a subharmonic function. This function satisfies the relations

$$
u(T z)=T u(z)
$$

and

$$
u(-r)=0 \quad \forall r \geq 0 .
$$

If $u(z)<r(1+\cos \theta)$ then $u$ is harmonic in some $\delta$-neighborhood of $z$; indeed, otherwise the function $u_{\delta}$ continuous in $\mathbb{C}$, coinciding with $u$ outside this neighborhood and harmonic inside, belongs to $\mathscr{U}_{\epsilon}$. The same argument shows that if $u \not \equiv 0$ then $u>0$ outside $(-\infty, 0] \cup \mathscr{R}_{\epsilon}(T, \tau)$. We will prove later that $u \neq 0$. Assuming this let us finish the proof of the lemma. If $\nu>0$ is small enough then

$$
u(z)<r(1+\cos \theta), \quad z=r e^{i \theta} \in I_{\epsilon+\nu},
$$

and, hence, $u$ is harmonic in $I_{\epsilon+\nu} \backslash \bar{I}_{\epsilon}$. Since $u$ is positive and harmonic in $I_{\epsilon+\nu} \backslash \bar{I}_{\epsilon}$ and $u(z)=0, z \in \bar{I}_{\epsilon}$, the normal derivative of $u$ (in the direction of outward normal to the boundary of $\bar{I}_{\epsilon}$ ) has positive infimum on $\partial \bar{I}_{\epsilon}$. Denote by $G_{I_{\epsilon}}(z, 1)$ the Green function of $I_{\epsilon}$ with the pole at 1 . We remark that its normal derivative is bounded since the boundary of $I_{\epsilon}$ is smooth. Hence, the function

$$
v(z)= \begin{cases}u(z) & \text { for } z \in \mathbb{C} \backslash \mathscr{R}_{\epsilon}(T, \tau), \\ -\gamma T^{k} G_{I_{\epsilon}}\left(z / T^{k}, 1\right) & \text { for } z \in T^{k} I_{\epsilon}\end{cases}
$$


is subharmonic if $\gamma>0$ is sufficiently small. It is clear that

$$
v(r) \leq-\alpha r, \quad r \in \mathscr{R}\left(T^{+}, \tau\right)
$$

for some positive $\alpha$.

The function

$$
g\left(r e^{i \theta}\right)=-r \cos \theta+v\left(r e^{i \theta}\right)
$$

satisfies all conditions of Lemma 1 due to properties (3)-(6).

It remains to prove that $u \not \equiv 0$ with an appropriate choice of $\epsilon$. Consider the domains

$$
\Omega_{\epsilon, \delta}=\left\{z:|\arg z|<\pi-\delta, z \notin \mathscr{R}_{\epsilon}^{+}(T, \tau)\right\}
$$

for some $\delta \geq 0$.

There exists a function $u_{\epsilon, \delta}$ positive and harmonic inside the domain $\Omega_{\epsilon, \delta}$ and vanishing on its boundary. This function is unique up to a multiplicative constant (see Appendix 1). As the region $\Omega_{\epsilon, \delta}$ is invariant under the transformation $z \mapsto T z$, we conclude from the uniqueness of $u_{\epsilon, \delta}$ that

$$
u_{\epsilon, \delta}(T z)=T^{\rho} u_{\epsilon, \delta}(z)
$$

for some positive $\rho=\rho(\epsilon, \delta)$. We always extend $u_{\epsilon, \delta}$ to a subharmonic function in the plane by putting it equal to zero outside $\Omega_{\epsilon, \delta}$. To complete the proof we need to show that for every fixed $T$ and $\tau \in(1, T)$ there exist $\epsilon$ and $\delta$ such that

$$
\rho(\epsilon, \delta)=1 \text {. }
$$

In this case the function $u_{\epsilon, \delta}(z)$ after multiplication by a suitable positive constant will belong to $\mathscr{U}_{\epsilon}$.

To prove $(8)$ we show that $\rho(\epsilon, \delta)$ is continuous for all admissible (so small that $\Omega_{\epsilon, \delta}$ is connected) values of $\epsilon$ and $\delta$ and takes values greater and smaller than 1 .

First, we show that $\rho(0,0)<1$. Indeed, $u_{0,0}$ is positive and harmonic in the upper halfplane. So

$$
\int_{\mathbf{R}} \frac{u_{0,0}(x)}{1+x^{2}} d x<\infty
$$

which is consistent with (7) only if $\rho<1$.

Second, it follows from the Phragmén-Lindelöf Principle that $\rho(\epsilon, \delta)>1$ if $\delta>\pi / 2$.

Since the continuity of $\rho(\epsilon, \delta)$ is proved in Appendix 1 , this completes the proof of Lemma 1.

We need also the following lemma.

Lemma 2. For every $\Delta \in(0,1)$ and $T>1$ there exist $\tau \in(1, T)$ and $a$ subharmonic function $v$ such that

$$
\begin{gathered}
v(-r)=B(v, r)=r^{\Delta} \text { for } r \in[0, \infty), \\
v(r)<-2 r^{\Delta} \quad \text { for } r \in \bigcup_{k=-\infty}^{\infty}\left[\tau^{-1 / \Delta} T^{k / \Delta}, T^{k / \Delta}\right] .
\end{gathered}
$$


Proof. Let $M=T^{1 / \Delta}$. Consider the function

$$
g(z)=\int_{0}^{+\infty} \log \left|1-\frac{z^{2}}{t^{2}}\right| d \mu(t),
$$

where $\mu$ is the measure supported by the set $\left\{M^{k}\right\}_{k=-\infty}^{\infty}$ and

$$
\mu\left\{M^{k}\right\}=M^{\Delta k}, \quad k \in \mathbb{N} .
$$

It is easy to see that integral (12) defines a subharmonic function with $g(M z)$ $=M^{\Delta} g(z), \quad z \in \mathbb{C}$. Now put

$$
f\left(r e^{i \theta}\right)=f\left(r e^{-i \theta}\right)=r^{\Delta} \cos (\Delta(\theta-\pi / 2)), \quad 0 \leq \theta \leq \pi .
$$

The function $f$ is subharmonic in the plane. Then the function

$$
u_{\gamma}(z)=\gamma g(z)+f(z)
$$

is subharmonic for every positive $\gamma$ and satisfies the relations

$$
\begin{gathered}
r^{\Delta}(1+\gamma A) \geq u_{\gamma}( \pm i r)=B\left(r, u_{\gamma}\right) \geq r^{\Delta}(1-\gamma A), \quad r \in \mathbb{R}, \\
u_{\gamma}(M z)=M^{\Delta} u_{\gamma}(z), \quad z \in \mathbb{C},
\end{gathered}
$$

where $A=\sup \left\{g(i r) / r^{\Delta}: r>0\right\}$ (it is clear that $0<A<\infty$ ). Setting

$$
\tilde{u}_{\gamma}\left(r e^{i \theta}\right)= \begin{cases}u_{\gamma}(z) & \text { for }|\theta| \leq \pi / 2, \\ \max \left\{B\left(r, u_{\gamma}\right),(1+A \gamma) r^{\Delta} \cos (\Delta(\pi-\theta))\right\} & \text { for }|\pi-\theta|<\pi / 2,\end{cases}
$$

we show that according to (13) this function is subharmonic if $\gamma>0$ is small enough. Indeed, if $\gamma>0$ is sufficiently small then the inequality

$$
(1+\gamma A) \cos (\Delta \pi / 2)<(1-\gamma A) \leq B\left(r, u_{\gamma}\right) r^{-\Delta}
$$

is valid, so $\tilde{u}_{\gamma}$ is equal to $u_{\gamma}$ in some angle $\left\{r e^{i \theta}:|\theta|<\delta\right\}, \delta>\pi / 2$, and $\tilde{u}_{\gamma}(-r)=B\left(r, \tilde{u}_{\gamma}\right)=r^{\Delta}(1+\gamma A)$.

Using (13), (14), and the equality $\tilde{u}_{y}(1)=-\infty$ we conclude that the function

$$
v(z)=(1+\gamma A)^{-1} \tilde{u}_{y}(z)
$$

satisfies all conditions of Lemma 2 for some suitable $\tau$. Lemma 2 is proved.

\section{CONSTRUCTION OF THE EXAMPLE}

Suppose $\rho \in(1,2)$, and let $\Delta=\rho-1$. We choose $T>1$ arbitrarily; for example, put $T=2$. According to Lemma 2 there exist $\tau \in(1, T)$ and a subharmonic function $v$ satisfying (10) and (11). According to Lemma 1 for this $\tau$ there exists a subharmonic function $g$ which satisfies (1) and (2) for some $\alpha>0$. Define the function $u$ in the following way:

$$
u\left(r e^{i \theta}\right)= \begin{cases}g\left(r^{1+\Delta} e^{i \theta(1+\Delta)}\right) & \text { for }|\theta| \leq \pi /(1+\Delta), \\ v\left(-r^{1+1 / \Delta} e^{i \theta(1+1 / \Delta)}\right) & \text { for }|\pi-\theta|<\pi \Delta /(1+\Delta) .\end{cases}
$$

It follows from (1) and (10) that this function $u$ is subharmonic in the plane and satisfies (ii). Property (iii) follows from (2) and (11).

For $\rho \geq 2$ consider $u\left(z^{n}\right)$ with a suitable $n \in \mathbb{N}$.

The example is constructed. 


\section{APPENDIX 1}

The results in this appendix are essentially known, but we could not find a convenient reference.

1. Let $\Omega=\Omega_{\epsilon, \delta}$ be the domain described in the proof of Lemma 1. We prove that there exists a function $u$ positive and harmonic in $\Omega$ and vanishing on $\partial \Omega$. Denote by $G$ the Green function of $\Omega$; fix a point $z_{0} \in \Omega$; and choose a sequence $z_{k} \rightarrow \infty, z_{k} \in \Omega$. Then the sequence of positive harmonic functions $u_{k}(z)=G\left(z, z_{k}\right) / G\left(z_{0}, z_{k}\right)$ is equicontinuous at every point $z \in \Omega$. If we extend $u_{k}$ by putting $u_{k}(z)=0, z \in \mathbb{C} \backslash \Omega$, then the sequence $u_{k}$ will be equicontinuous in $\bar{\Omega}$. This follows from a simple application of the twoconstants theorem. Thus any limit function $u$ of the sequence $u_{k}$ is positive and harmonic in $\Omega$ and vanishes on the boundary. We have $u\left(z_{0}\right)=1$, so $u \not \equiv 0$.

2. Let us prove that a positive harmonic function in $\Omega$ vanishing on the boundary is unique up to a multiplication by a positive constant. We use a modification of the argument in [Kj].

Let $U$ be the set of all such functions. Without loss of generality we may suppose that $\delta$ in the definition of the domain $\Omega$ is equal to $\pi / 2$ since the general case may be reduced to this by conformal mapping.

Let $K=\left\{r_{0} e^{i \theta}:|\theta| \leq \pi / 2\right\}, r_{0} \in \Omega$. Let $u \in U$. It is easy to see that the function $g(z)=u(z) / \Re z$ defined in the right halfplane can be extended continuously to $K$ and has a positive infimum on $K$.

The basic assertion that we will use for the proof of the uniqueness is the following. There exists $d>0$ such that for every $u \in U$ we have

$$
\frac{\max _{z \in K} g(z)}{\min _{z \in K} g(z)} \leq d .
$$

Assume that $(15)$ is false. Then there exists a sequence $u_{k}(z) \in U$ such that

$$
\frac{\max _{z \in K} g(z)}{\min _{z \in K} g(z)}>k \text {. }
$$

We may suppose $B\left(2 r_{0}, u_{k}\right)=1$. By the Harnack inequality the sequence $\left\{u_{k}\right\}$ is a precompact family of harmonic functions on $\Omega$, and by normalization no subsequence of this sequence tends to $\infty$ or to identical 0 . Applying the twoconstants theorem we see that all limit functions vanish on the boundary, so all limit functions belong to $U$. So (16) cannot occur and (15) holds.

We remark that (15) remains true (with the same constant $d$ ) if we take $z \in$ $T^{k} K$ instead of $z \in K$ since $U$ is invariant with respect to the transformations $u(z) \mapsto u\left(T^{k} z\right)$.

Let $u, v \in U$ be two different functions. Let $\alpha_{k}$ be the sequence defined as

$$
\alpha_{k}=\sup \left\{\alpha: u(z)-\alpha_{k} v(z) \geq 0 \text { for } z \in T^{k} K\right\}
$$

It is evident that $\alpha_{k} \searrow \alpha$ for some nonnegative $\alpha$. Then set $\eta_{k}=\alpha_{k}-\alpha \rightarrow 0$. The sequence

$$
u(z)-\alpha_{k} v(z)=r_{k}(z)
$$

converges to a nonnegative function $r(z)=r_{k}(z)+\eta_{k} v(z)$, and either $r(z) \in U$ or $r(z) \equiv 0$. According to the definition of $\alpha_{k}$ the function $r_{k}(z) / \Re z$ is zero 
at some point of $T^{k} K$, since otherwise (15) implies that $\left(r_{k}(z)-\eta v_{k}(z)\right) / \Re z$ will be positive on $T^{k} K$ for some $\eta>0$. Thus we have

$$
\frac{r\left(z_{k}\right)}{\Re z_{k}}=\eta_{k} \frac{v\left(z_{k}\right)}{\Re z_{k}}
$$

for some point $z_{k} \in T^{k} K$. Therefore, applying (15) to both the left and right sides of the last relation we obtain

$$
\frac{r(z)}{\Re z}<d^{2} \eta_{k} \frac{v(z)}{\Re z}
$$

for every point $z \in K$. (This proof is valid when $r(z) \in U$. When $r(z) \equiv 0$ this inequality is trivial.) By the maximum principle

$$
r(z)<d^{2} \eta_{k} v(z) \quad \text { for }|z|<T^{k} K ;
$$

hence $r(z) \equiv 0$. So we get $u(z)=\alpha v(z)$, and hence the uniqueness is proved.

3. Finally we prove that the order $\rho(\epsilon, \delta)$ of the positive harmonic function $u_{\epsilon, \delta}$ in $\Omega_{\epsilon, \delta}$ vanishing on the boundary is continuous for $0 \leq \delta \leq \pi / 2$ and $0 \leq \epsilon \leq \epsilon_{0}$, where $\epsilon_{0}$ is small enough. It follows from the uniqueness of $u_{\epsilon, \delta}$ that $u_{\epsilon, \delta}(T z)=C u_{\epsilon, \delta}(z)$, where $C=T^{\rho}$. So

$$
\rho(\epsilon, \delta)=\frac{\log \left(u_{\epsilon, \delta}\left(T z_{0}\right) / u_{\epsilon, \delta}\left(z_{0}\right)\right)}{\log T},
$$

where $z_{0}$ is an arbitrary point in $\Omega_{\epsilon, \delta}$. Assume that $\epsilon_{n} \rightarrow \epsilon_{0}, \delta_{n} \rightarrow \delta_{0}$. We may suppose that $z_{0} \in \Omega_{\epsilon_{n}, \delta_{n}}$ for all $n$. Then (after selection of a subsequence if necessary) we have $u_{\epsilon_{n}, \delta_{n}} \rightarrow v$, on compacta in $\Omega_{\epsilon_{0}, \delta_{0}}$, where $v$ is a positive harmonic function in $\Omega_{\epsilon_{0}, \delta_{0}}$, vanishing on the boundary. From the uniqueness it follows that $v=C u_{\epsilon_{0}}, \delta_{0}$, and we have

$$
\rho\left(\epsilon_{n}, \delta_{n}\right) \rightarrow \rho\left(\epsilon_{0}, \delta_{0}\right)
$$

in view of (17).

\section{APPENDIX 2}

In this appendix we show how to get the corresponding entire example from the subharmonic one. We need an approximation theorem of Azarin [A] and the notion of a $C_{0}$-set.

First we give the definition of a $C_{0}$-set. A set $E$ is called a $C_{0}$-set if it may be covered by disks $B\left(\rho_{k}, \xi_{k}\right)$ ( $\rho_{k}$ are their radii, $\xi_{k}$ are the centers) such that the relation

is valid.

$$
\lim _{r \rightarrow \infty} \frac{1}{r} \sum_{\left|\xi_{k}\right| \leq r} \rho_{k}=0
$$

Now we cite Azarin's theorem on the approximation of subharmonic functions.

Theorem A. Let $u(z)$ be a subharmonic function of finite proximate order $\rho(r)$. Then there exists an entire function $f(z)$ of the same proximate order $\rho(r)$ and the same type with respect to this order, and there exists a $C_{0}$-set $E$ such that the estimate

holds outside $E$.

$$
\log |f(z)|-u(z)=o\left(|z|^{\rho(|z|)}\right), \quad|z| \rightarrow \infty,
$$


Let $D$ be an open set intersecting every circle $|z|=r$ such that $T D=D, T$ being taken from the definition of the subharmonic function $u(z)$. Let $E$ be a $C_{0}$-set. It is easy to see that there exists $r_{0}$ such that the set $D \backslash E$ intersects every circle of radii larger than $r_{0}$. Indeed, if this is not so then there exists a sequence $r_{k} \rightarrow \infty$ such that

$$
\operatorname{mes}\left\{\varphi: r_{k} e^{i \varphi} \in E\right\} \geq \operatorname{mes}\left\{\varphi: r_{k} e^{i \varphi} \in D\right\} \geq \delta>0, \quad r_{k} \rightarrow \infty .
$$

But the latter inequality contradicts the definition of a $C_{0}$-set.

Now taking $\epsilon>0$ small enough to satisfy $1+\alpha-\epsilon>1$ and setting $D=$ $\left\{z: u(z)<-(1+\alpha-\epsilon)|z|^{\rho}\right\}$ we can construct by Azarin's theorem an entire function $f(z)$ and a $C_{0}$-set $E$ such that

$$
B(|z|, \log |f(z)|)-B(|z|, u(z))=o\left(|z|^{\rho}\right), \quad|z| \rightarrow \infty, \quad z \in \mathbb{C},
$$

and

$$
\log |f(z)|-u(z)=o\left(|z|^{\rho}\right), \quad|z| \rightarrow \infty, \quad z \in \mathbb{C} \backslash D .
$$

Since the set $D \backslash E$ intersects every circle $|z|=r$ for all sufficiently large $r>r_{0}$, we have

$$
B(|z|, \log |f(z)|)=(1+o(1))|z|^{\rho}, \quad|z| \rightarrow \infty,
$$

and

$$
L(|z|, \log |f(z)|)<-(1+\alpha-\epsilon)(1+o(1))|z|^{\rho}, \quad|z| \rightarrow \infty,
$$

and hence the function $f(z)$ gives the needed example.

\section{ACKNOWLEDGMENT}

I thank David Drasin, Alex Eremenko, and Mike Sodin for stimulating discussions and numerous corrections in the manuscript.

\section{REFERENCES}

[A] V. S. Azarin, On asymptotic behavior of subharmonic function of finite order, Math. USSRSb. 36 (1980), 135-154.

[Kj] Bo Kjellberg, On certain integral and harmonic functions, Thesis, Uppsala, 1948.

[H1] W. K. Hayman, Research problems in function theory, Symposium on Complex Analysis (Canterbury, 1973), Cambridge Univ. Press, Cambridge, 1974, pp. 155-180.

[H2] The minimum modulus of large integral function, Proc. London Math. Soc. 2 (1952), 469-512.

[Yu] R. S. Yulmukhametov, The approximation of subharmonic functions, Anal. Math. 11 (1985), 257-282.

Division of Mathematics, Institute for Low Temperature Physics and Engineering, 47 Lenin Avenue, Kharkov 310164, Ukraine

E-mail address: fryntov@ilt.kharkov.ua 